\section{Prophylactic TNF blockade reduces autoimmune toxicity}

Despite the synergistic efficacy of nivolumab and ipilimumab in some advanced-stage solid tumours, serious immune-related adverse events (irAEs) are common. A new study now demonstrates that prophylactic tumour necrosis factor (TNF) blockade reduces gastrointestinal irAEs in mice treated with dual immune-checkpoint inhibition (DCI).

The group of Ignacio Melero (University of Navarra, Spain) first demonstrated that DCI exacerbated dextran sulfate sodium (DSS)-induced autoimmune colitis in mice, which was ameliorated with prophylactic administration of an anti-TNF antibody or the TNF inhibitor etanercept. Importantly, TNF blockade before DCI did not impair antitumour efficacy in syngeneic mouse models of colon cancer and melanoma, and even improved the survival and xenograft-rejection rate in DSS-treated syngeneic mouse models.

TNF blockade was found to enhance DCI-induced $\mathrm{CD}^{+} \mathrm{T}$ cell infiltration (in the tumour and lymph nodes) and decrease activation-induced cell death in DCI-treated $\mathrm{CD}^{+} \mathrm{T}$ cells in vitro and in vivo, providing a mechanistic rationale for these observations.

The expression of TNF-related transcripts and genes was found to be upregulated in normal colonic mucosal tissues from a group of four patients treated with DCI who developed colitis compared with those from four healthy individuals without bowel inflammation or cancer. In a graft-versus-host disease mouse model, prophylactic etanercept treatment reduced DCI-induced

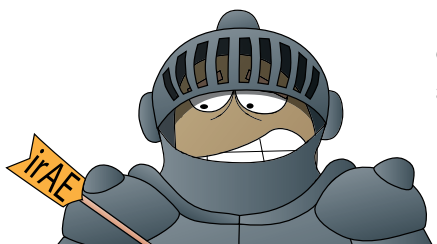

exacerbation of colitis. Similarly, in a colon cancer xenograft-induced humanized mouse model of colitis, concomitant etanercept treatment did not weaken the antitumour effects of nivolumab-ipilimumab, and, importantly, it diminished xenograft-induced colitis.

The findings have clear translational relevance, whereby prophylactic TNF inhibition could improve the safety of DCI regimens while Credit: Philip Patenall/ Springer Nature Limited preserving - or even

prophylactic TNF inhibition could improve the safety of $\mathrm{DCl}$ regimens while preserving - or even enhancing efficacy

5
enhancing - efficacy, and perhaps enabling ipilimumab doses to be safely increased.

"An ongoing pilot trial in France (NCT03293784) is testing safety of the combined strategy, and a larger randomized phase II trial should soon test efficacy," adds Melero.

Conor A. Bradley

\title{
Gene-fusion neoantigens stimulate T cells
}

Tumours with high somatic mutation burden typically respond better to immune checkpoint inhibitors. However, responses are sometimes observed in patients with a low mutational burden. Yang et al. identified an exceptional responder with metastatic head and neck squamous cell carcinoma (HNSCC) who exhibited complete response to anti-PD1 immunotherapy despite a low mutational burden, and demonstrated that gene fusions were a source of neoantigens in this patient and others.

Whole-genome sequencing and RNA sequencing identified a novel $D E K-A F F 2$ gene fusion. To determine the mechanisms associated with the response, they used a machine-learning approach to test peptides derived from both mutations and gene fusions in the tumour, and found a DEK-AFF2 fusion-derived peptide that stabilized

patient-specific HLAs. Expression of DEK-AFF2 led to a cytotoxic response

the findings

... could pave

the way for novel immunotherapies for other cancers ... with ... low mutational burden

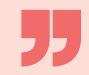
in the patient's T cells against an HNSCC cell line, and a T cell clone associated with DEK-AFF2 was identified in the patient's blood using T cell receptor sequencing, suggesting that the response to anti-PD1 treatment was most likely attributable to the DEK-AFF2 fusion.

To extend this to other cancers, the authors looked at 20 head and neck adenoid cystic carcinomas - a cancer type associated with low tumour mutational burden, low immune infiltration and prevalent MYB-NFIB gene fusions. Here, they also identified gene-fusion-derived antigens that elicited cytotoxic T cell responses.

The authors used RNA sequencing data for 30 cancer types with gene fusions from The Cancer Genome Atlas to examine neoantigen presence and its relationship with the immune microenvironment. Of the tumours examined, $24 \%$ had a fusion neoantigen predicted to bind to a patient-specific HLA, and there was an inverse relationship between immune cell infiltration and the presence of a fusion neoantigen, suggesting that immunoediting might occur. Indeed, in a cohort of patients with melanoma a comparison of biopsy samples taken both before and during anti-PD1 treatment showed that there was a reduction in the number of fusion neoantigens during immunotherapy, which is possibly indicative of immunoediting against gene-fusion-derived neoantigens.

The authors hope that the findings, which demonstrate that gene fusions are a source of tumour-specific neoantigens, could pave the way for novel immunotherapies for other cancers associated with gene fusions, low mutational burden and an unfavourable immune environment.

Jordan Hindson

ORIGINAL ARTICLE Yang, W. et al. Immunogenic neoantigens derived from gene fusions stimulate T cell responses. Nat. Med. 25, 767-775 (2019) 